\title{
Robust features for textures in additive noise
}

\author{
C.Ottonello ${ }^{1}$, S.Pagnan ${ }^{2}$ and V. Murino ${ }^{1}$ \\ ${ }^{1}$ Dip. di Ingegneria Biofisica ed Elettronica-University of Genoa \\ Via all'Opera Pia 11A, 16145 Genova, Italy \\ 2 Istituto di Automazione Navale- National Research Council of Italy \\ Torre di Francia, Via De Marini 1, 16149 Genova, Italy
}

\begin{abstract}
The paper describes a method for texture classification in noise by using thirdorder cumulants as discriminating features. The problem is formulated as a test on $\mathrm{K}$ hypotheses and solved by a Maximum Likelihood (ML) criterium applied in the third-order cumulant domain. Since in the case of image processing complete third-order cumulant computation is not feasible, we reduced the estimation to a limited number of cumulant slices and lags. This reduction makes the classification algorithm suboptimal. Thus, a criterion for the choice of cumulant samples to be computed is introduced in order to guarantee the selection of those lags which better identify the different textures in the training phase of the classifier.

Experimental tests are carried out to evaluate third-order cumulant performances on noisy textures and the importance of lags selection.
\end{abstract}

\section{Introduction}

The aim of texture classification algorithms is to produce a set of measures that make it possible to discriminate between different classes of textures so that each class may be described by parameters that can be used by a segmentation algorithm to partition an image into homogeneous regions. When images are affected by noise, this purpose can be strongly compromised and it is necessary to produce robust measures for each class of texture. In the paper, a Maximum Likelihood (ML) classifier working in the domain of Higher Order Statistics (HOS) [1] is proposed; in particular, third-order cumulants are estimated as texture features, thanks to their insensitivity to symmetric and independent, identically distributed (i.i.d.) noises. In order to reduce computation efforts and to determine an appropriate set of cumulant samples to be included in the feature vector, the number of outliers for each class is evaluated during the training phase. Cumulant samples inconsistent with the expectation produced by each class population are ranked out by assigning them small efficiency weights.

In section 2, the classifier scheme is presented, and, in subsection 2.1, the adopted criterion for lag selection is discussed. Section 3 describes the experiments performed on natural textures corrupted by i.i.d. as well as coloured Gaussian noise; in this section, a comparison with an autocorrelation based classifier is also made. Some conclusions are carried out in section 4 . 


\section{The Classifier}

The problem of classifying signals corrupted by noise can be reduced to a test of $\mathrm{K}$ hypotheses, and, for additive noise, can be expressed as:

$$
H_{k}: x(i)=n(i)+s_{k}(i) \quad \mathrm{i}=\left(\mathrm{i}_{1}, \mathrm{i}_{2}\right)=1 \ldots \mathrm{R} ; \mathrm{k}=1 \ldots \mathrm{K}
$$

where $\mathrm{n}(\mathrm{i})$ denotes noise samples, $s_{k}(i)$ is the image template belonging to the class $k, x(i)$ are observation samples, and $i=\left(i_{1}, i_{2}\right)$ are the pixel indexes.

As alternative to energy classifiers/detectors, schemes based on higher-order statistics have been proposed, which are insensitive to additive Gaussian noise and have the characteristic of preserving phase information (i.e., they exhibit sensitivity to signal shape) [2] [3]. In (1) the observation vector $x(i)$ can be replaced by the Nxl vector $\hat{f}_{N}$, which contains all estimated HOS samples and the ML classifier can be implemented, as follows:

$$
\ln p\left(\hat{f}_{N} / H_{k}\right)_{<H_{l}}^{H_{k}} \ln p\left(\hat{f}_{N} / H_{l}\right)
$$

From [4], it derives that such probabilities are normal.

In the proposed classification scheme, in order to obtain a classifier robust to a large class of noises, we replace $\hat{f}_{N}$ with $\mathrm{N}$ lags of the third-order cumulant, that is defined for zero-mean signals as:

$$
\hat{c}_{3}^{x}\left(\tau_{1}, \tau_{2}\right)=E\left\{x(i) x\left(i+\tau_{1}\right) x\left(i+\tau_{2}\right)\right\}
$$

where we assume $\tau_{1}=\left(\tau_{11}, \tau_{12}\right)$ and $\tau_{2}=\left(\tau_{21}, \tau_{22}\right)$ as the spatial lags in the two image directions, and reduce test (2) to a minimum-distance or minimum-HOSenergy classifier (by setting the covariance matrix $\hat{\Sigma}\left({ }_{H_{k}}\right)=\mathrm{I}$ ). We choose the hypothesis $H_{k}$

$$
\text { iff }\left|\hat{c}_{3}^{x}-\bar{c}_{3}\right|^{2}<\left|\hat{c}_{3}^{x}-\bar{c}_{3}\right|^{2}
$$

Due to computational efforts, only some cumulant lags can be computed in practice, thus exploiting the HOS potentiality only partially. These drawbacks make HOS performances comparable to those of energy-based classifiers, in some practical cases where few samples are available.

Within the context, a considerable improvement can be obtained by performing the classification process after selecting the most effective lags for discrimination [3].

\subsection{Feature Selection}

From an initial set of lag samples, estimated on noise-free images, $\mathrm{N}$ lags can be selected to obtain an appropriate feature vector by assigning larger penalties to lags 
with larger numbers of outliers and interclass-overlapping regions, according to the following scheme:

$$
W\left(\text { lag }_{i}\right)=\sum_{k=1}^{K} \text { ouliers }\left(\text { lag }_{i}, \text { class }_{k}\right)+\sum_{k=1}^{K} \sum_{j=k+1}^{K} \delta\left\{C_{k}^{i} \cap C_{j}^{i}\right\}
$$

where outlier $\left(\operatorname{lag}_{\mathbf{j}}\right.$, class $\left.{ }_{k}\right)$ is a cumulant sample outside the range $\left[m_{k}^{i}-2 \cdot \sigma_{k}^{i}, m_{k}^{i}+2 \cdot \sigma_{k}^{i}\right]$ for cumulant sample $\mathrm{i}$ of class $\mathrm{k}, \mathrm{C}_{\mathrm{k}}^{\mathrm{i}}$ is the set including all estimated lags i for class $\mathrm{k}$, and $\delta\left\{C_{k}^{i} \cap C_{j}^{i}\right\}=1$ if $C_{k}^{i} \cap C_{j}^{i} \neq \varnothing$ and it is equal to 0 , otherwise.

\section{Experimental Tests}

Test Case \#1: The classification algorithm defined by relation (4) was applied to the first image set (Fig.1). Each 256×256 image was divided into several subimages. I.i.d. Gaussian and coloured Gaussian noises were superimposed upon each sub-image. Coloured noise was obtained as the output of a linear filter driven by i.i.d. Gaussian noise. The leave-one-out strategy was adopted for the classification process. Each sub-image was presented to the classifier, after training it by using the remaining noise-free images. The classification probability was evaluated by using 50 noise realizations for different SNR values (calculated as

$10 \cdot \log 10\left\{E\left[s^{2} / n^{2}\right]\right\}$ ) for each test. The classification results obtained by the third-order cumulants, according to the minimum-distance criterion, were compared with autocorrelation results. In the absence of noise, $100 \%$ classification results were obtained by both classifiers. $\hat{c}_{3}^{x}$ yielded better results than $\hat{c}_{2}^{x}$ on noisy images, thanks to the insensitivity of the former cumulant to noise with zero skewness (Tables 1 and 2).

In both experiments, a limited number of lags were used. For $\hat{c}_{3}^{x}$, the feature vector was the set $\left\{c_{3}^{x}\left(\tau_{11}, \tau_{12} ; \tau_{21}, \tau_{22}\right): \tau_{11}=0 ; \tau_{12}=0,1 ; 0 \leq \tau_{21}, \tau_{22} \leq 3\right\}$.

For $\hat{c}_{2}^{x}$, the feature vector included $\left\{c_{2}^{x}(\tau): \tau=0, \ldots, 5\right\}$.

Then lag selection for $\hat{c}_{3}^{x}$ was performed to reduce the number of parameters necessary for an efficient classification. According to (5), the first $\mathrm{N}$ lags were extracted from the initial set:

$\left\{c_{3}^{x}\left(\tau_{11}, \tau_{12} ; \tau_{21}, \tau_{22}\right): \tau_{11}=0 ; \tau_{12}=0 ; 0 \leq \tau_{21}, \tau_{22} \leq 20\right\}$ 
estimated on clean images. Improvements obtained in classification results are shown in Table 3.

Test Case \#2: A new image set (Fig. 2) was tested to evaluate $\hat{c}_{3}^{x}$ insensitivity when i.i.d. is replaced with coloured noise. Unlike the previous test, lag selection was also performed for $\hat{c}_{2}^{x}$ samples in the set $\left\{c_{2}^{x}(\tau): \tau=0, \ldots, 19\right\}$. Although $\hat{c}_{2}^{x}$ performances were strongly improved by eliminating lag outliers, $\hat{c}_{2}^{x}$ sensitivity to coloured noise was verified. Figure 3 compares the $\hat{c}_{2}^{x}$ and $\hat{c}_{3}^{x}$ probability of correct classification in the presence of i.i.d. Gaussian noise and coloured Gaussian noise, for different SNRs and different numbers of features $(N)$. $\hat{c}_{2}^{x}$ classifier resultsed completely inneficient in the presence of coloured noise, whereas $\hat{c}_{3}^{x}$ performances remained unchanged.
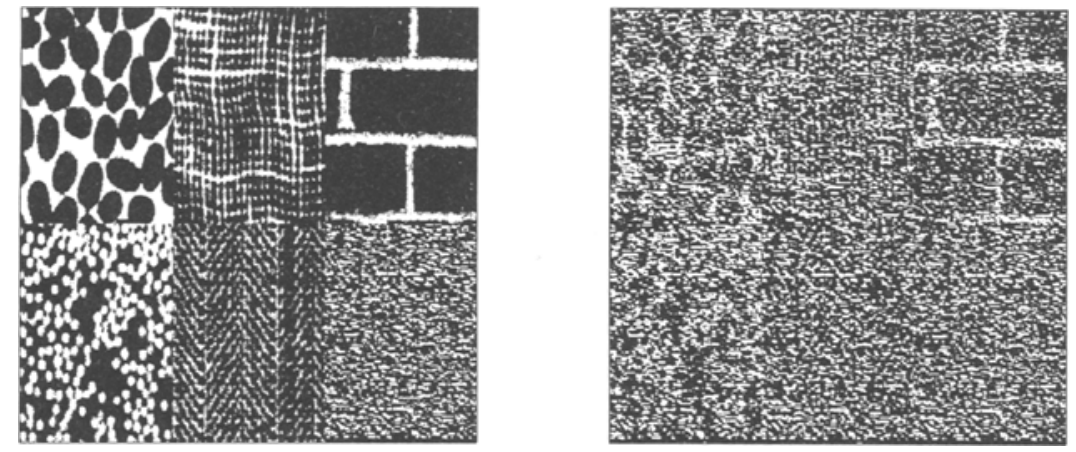

Fig.1: On the right: five original textures of test set \#1: coffee, cloth, wall, naphtha, tweed, and synthetic noise. On the left: test set \#1 corrupted by coloured Gaussian noise $(\mathrm{SNR}=-10 \mathrm{~dB})$.

\section{Conclusions}

An image classification scheme that exploits cumulant features has been proposed. The HOS reduced sensitivity to i.i.d. and coloured Gaussian noise and the capability of characterizing non-Gaussian signals justify the use of HOS in classification problems. The adopted cumulant-based method, even though suboptimal, is computationally efficient and yields good results, especially for very noisy images. A considerable improvement in classification results can be obtained by performing a cumulant sample selection, i.e. by removing from the feature set all samples that may lead to class overlapping. 
Results show that the proposed classification features are robust to very critical signal-to-noise ratios (SNRs); in particular, a correct classification is invariant to both i.i.d. and coloured Gaussian noise. Moreover, combination of third and second-order statistics can provide effective parameters for a noise-robust segmentation algorithm.

Table 1: Probabilities of correct classification ( 32 lags of $\hat{c}_{3}^{x}$ )

\begin{tabular}{|c|c|c|}
\hline SNR (dB) & $\begin{array}{c}\text { Gaussian } \\
\text { Noise }\end{array}$ & $\begin{array}{c}\text { Coloured } \\
\text { Gaussian } \\
\text { Noise }\end{array}$ \\
\hline-15 & .611 & .481 \\
\hline-10 & .859 & .748 \\
\hline-5 & .961 & .934 \\
\hline 0 & .977 & .974 \\
\hline 5 & .992 & .986 \\
\hline
\end{tabular}

Table 2: Probabilities of correct classification (36 lags of $\hat{\mathbf{c}}_{2}^{\mathrm{x}}$ )

\begin{tabular}{|c|c|c|}
\hline SNR (dB) & $\begin{array}{c}\text { Gaussian } \\
\text { Noise }\end{array}$ & $\begin{array}{c}\text { Coloured } \\
\text { Gaussian } \\
\text { Noise }\end{array}$ \\
\hline-15 & .200 & .200 \\
\hline-10 & .304 & .220 \\
\hline-5 & .989 & .950 \\
\hline 0 & 1 & 1 \\
\hline 5 & 1 & 1 \\
\hline \multicolumn{3}{|c}{} \\
\hline
\end{tabular}

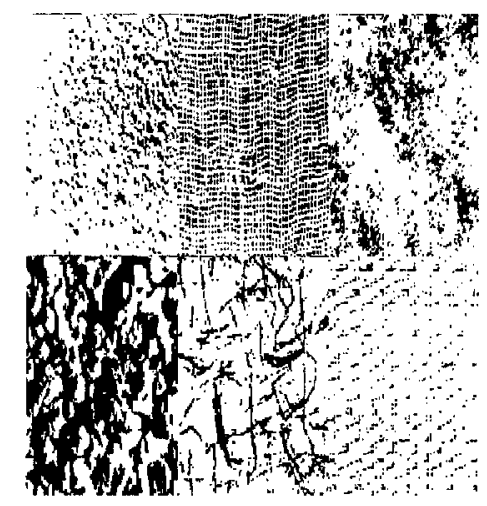

Fig. 2: Test set \#2. From left to right From left to right and from top to bottom: cork, gauze, ice, mica, ricepaper and wicker. 
Table 3: Probabilities of correct classification (N selected lags of $\hat{c}_{3}^{x}$ )

\begin{tabular}{|c|c|c|c|c|c|c|}
\hline SNR & \multicolumn{3}{|c|}{ Gaussian Noise } & \multicolumn{3}{c|}{ Coloured Gaussian Noise } \\
\hline $\mathrm{dB}$ & $\mathrm{N}=10$ & $\mathrm{~N}=20$ & $\mathrm{~N}=30$ & $\mathrm{~N}=10$ & $\mathrm{~N}=20$ & $\mathrm{~N}=30$ \\
\hline-15 & .445 & .5 & .64 & .39 & .51 & .59 \\
\hline-10 & .775 & .83 & .9 & .725 & .85 & .87 \\
\hline-5 & .94 & .97 & .955 & .915 & .96 & .96 \\
\hline 0 & .995 & .985 & .975 & .995 & .985 & .975 \\
\hline 5 & 1 & .995 & .98 & 1 & 1 & .98 \\
\hline
\end{tabular}

c2
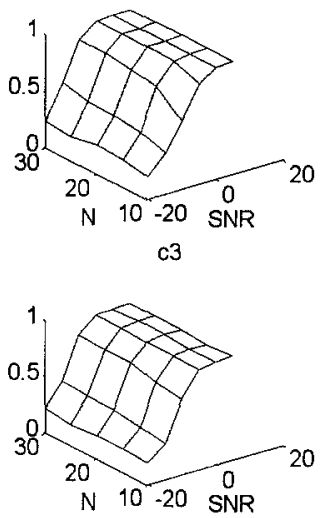

c2
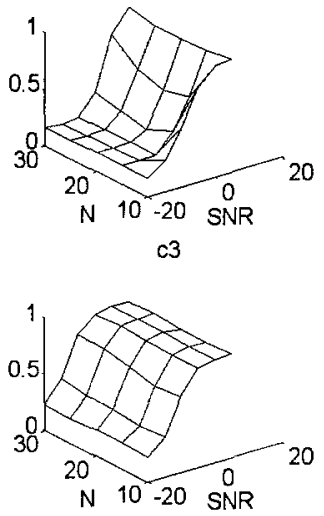

Fig. 3: Classification probabilities in the presence of i.i.d. (left) and coloured (right) Gaussian noises vs. SNR and number of lags, for $\hat{c}_{2}^{x}$ and $\hat{c}_{3}^{x}$.

\section{References}

[1] C.L. Nikias and J.M. Mendel: Signal processing with higher-order spectra. IEEE Signal Processing Magazine, pp. 10-37 (1993).

[2] G.B. Giannakis and M.K. Tsatsanis: A unifying maximum-likelihood view of cumulant and polyspectral measures for non-Gaussian signal classification and estimation. IEEE Trans. on Information Theory, 38, pp. 386-406 (1992).

[3] A. Makovec, and G. Ramponi: Supervised Discrimination of noisy textures using thirdorder moments. Fourth COST WG.1 and 2nd Workshop on Adaptive Methods and Emerging Techniques for Signal Processing and Communications, Slovenia, April 1994.

[4] D.R. Brillinger and M. Rosenblatt: Asymptotic theory of estimates of kth-order spectra. In: Spectral Analysis of Time Series, B.Harris, ed., Wiley, NY, pp.153-188, (1967). 